\title{
La Universidad y la Ruptura con los Saberes Profesionalizantes
}

\author{
Eduardo López Herrera ${ }^{1}$
}

\section{RESUMEN}

La universidad, en el actual contexto contemporáneo, tiene una enorme responsabilidad frente a los retos que plantea el siglo XXI, por lo tanto, este ensayo quiere invitar a la reflexión sobre qué modelo de universidad necesitamos construir, de forma tal, que podamos romper con saberes tradicionales que ya no tienen cavidad en este nuevo siglo y que, de no hacerlo, las máximas casas de estudio quedaran relegadas y superadas por lo que muchos entendidos llaman la post universidad. Aprender para transformar debe ser un eje fundamental en la universidad del siglo XXI, pero aprendizajes con pertinencia y no profesionalizantes, porque estos últimos son una rémora que no abonan a los nuevos paradigmas que demanda la sociedad del aprendizaje permanente que hoy se vive, que además, está signada por las tecnologías de información y comunicación que viabilizan un componente virtual que no se puede obviar siempre y cuando sea una virtuosidad real en función de una educación de calidad. Romper con los saberes profesionalizantes, es darle cabida a la innovación y el emprendimiento, como dos ejes claves de la educación del siglo XXI y que la universidad los debe potenciar a partir de la gestión del conocimiento que solo se logra a través de la investigación como el quehacer pedagógico de la universidad.

Recibido: 5 de septiembre de 2017

Aceptado: 25 de noviembre de 2017

1 Miembro del Consejo de Facultad. FAREM Estelí de UNAN-Managua. Correo elctrónico: edujlopez9@gmail.com 


\title{
The university and the rupture with professional knowledge
}

\begin{abstract}
The university in the current contemporary context, has a big responsibility in facing the challenges that are presented in the XXI century, Therefore, the aim of this essay is to make you reflect about the university model that we need to construct, in that way, traditional knowledge can be taken away since they do not accommodate to this new century, if not, these prestigious universities will be relegated and exceeded by what many experts call the post university. Learning to transform must be a fundamental axis in the XXI century university, but with appropriateness and non-professional, because the latter are a difficulty that do not contribute to the new paradigms demanded by the society of Long-term learning that is lived today, which also, is marked by the information and communication technologies that make possible a virtual component that can not be ignored as long as it is a real virtuosity based on a quality education. Break with professional knowledge, is to accommodate innovation and entrepreneurship, as two key axes of education in the XXI century and that university must empower based on knowledge management that is only achieved through research as the pedagogical task of the university.
\end{abstract}

\section{INTRODUCCIÓN}

El contexto en el cual se mueve la universidad, hoy en día, es muy dinámico; determinado por los nuevos desafíos, por los nuevos retos que impone el mundo contemporáneo, signado por los cambios vertiginosos del conocimiento. La universidad es una institución que por su carácter educativo universal, por ser un centro de difusión de ciencia y de cultura, ha dejado su marca indeleble en cada contexto histórico en que le ha tocado estar desde que es universidad y los cambios y su contribución al desarrollo de la nación han sido claves, por ejemplo; en 1860, encontramos una universidad que ha incluido en sus programas: gramática castellana, matemática, inglés, francés; ya se iban preparando las condiciones para insertarnos en la división internacional del trabajo que el Capitalismo establecía, también respondía a un proceso de organización sobre la base del modelo napoleónico o profesionalizante, bajo la influencia del esquema organizativo asumido por el resto de universidades latinoamericanas.

Otro ejemplo importante en la evolución de la universidad pública nicaragüense, se produjo con los cambios en la educación demandados por el cultivo del algodón, esto implicó adaptarse a un contexto en que el Capitalismo dependiente no requería de un conglomerado estrictamente tecnológico, por lo que el perfil de nuestra Alma Mater se enfocó hacia una universidad de servicio, formadora de abogados, administradores, tenedores de libros, etc. 
El peso de la universidad napoleónica ha estado presente hasta el día de hoy, es decir, los saberes profesionalizantes son una rémora que no pueden seguir siendo el eje del quehacer educativo, cuando los cambios que la sociedad del siglo XXI demanda, van más allá de los simples saberes; se trata de una sociedad del conocimiento que demanda: investigación, innovación y emprendimiento, de manera tal que los saberes sean pertinentes, que sean producto de una renovación constante, de creatividad académica, que facilite procesos de inserción en un mundo que reclama nuevas formas de producción, nuevos modelos de formación; iniciativas novedosas para gestionar conocimiento, en fin; una universidad empoderada de una visión cultural y científica del mundo que se pone al frente de un cambio paradigmático como es romper con una praxis profesionalizante y hacer de su quehacer una innovación dialéctica como aporte sustantivo a una teoría universitaria contemporánea, pertinente y forjadora de cambios estructurales en el pensamiento, en la naturaleza y en la sociedad.

\section{La Universidad: entre la continuidad y el cambio}

La disyuntiva que hoy enfrenta la universidad, hay que considerarla muy responsablemente, porque se trata de vivir o morir, visto desde la perspectiva de un mundo cambiante y exigente con la ciencia para que respalde esos cambios, no obstante; se trata de ciencia actualizada, ya no del conocimiento de base que tuvo en su momento su justificación y que los saberes siempre lo tuvieron como referencia académica y científica. Hoy en día, ese conocimiento de base es una referencia epistemológica como acervo de cultura científica. Insignes pensadores han dejado una huella muy visible como constructores del mismo, veamos: "Dame un punto de apoyo y moveré el mundo" dijo Arquímedes, "Pienso, luego existo” decía Renato Descartes y de esa manera sentaba las bases de la ciencia moderna. "Lo que sabemos es una gota de agua, lo que ignoramos es el océano”, nos decía Isaac Newton; no obstante, esa base es un punto de referencia que cumplió su cometido, en tanto que la misma ha evolucionado a estadios más complejos y sofisticados, caracterizándolo además; su avance vertiginoso, su renovación constante, al respecto, el futurólogo de Google, Ray Kurzweil, indica que: “... Un período futuro durante el cual el ritmo del cambio tecnológico será tan rápido y su impacto tan profundo, que la vida humana se transformará irreversiblemente. Aunque ni utópica ni distópica, esta época va a transformar los conceptos en los que nos apoyamos para dar sentido a nuestras vidas, de nuestros modelos de negocio para el ciclo de la vida humana, incluyendo la muerte”.

¿Está la universidad preparada para ser parte de los cambios que demanda esta sociedad del conocimiento y del aprendizaje permanente? No tiene otra alternativa que hacerlo y la vía más expedita es a través de procesos de innovación donde el I-D-I es fundamental para que la universidad contribuya gestionando nuevos conocimientos, que se traduzcan en aportes científico técnicos al servicio de la sociedad y esto sólo será posible con una concepción y una visión prospectiva que rompa definitivamente con el homo académicus todavía presente y proponga un cambio paradigmático en su quehacer educativo para que responda a las demandas y necesidades 
de un siglo XXI muy exigente en el campo del conocimiento y su aplicación y la universidad no puede estar al margen de esa exigencia.

Y como señala Philip Coombs: "Las universidades no tiene otra opción real sino atender nuevas necesidades porque, si dejan de hacerlo, se hallarán otras maneras de atender estas demandas y las universidades que funcionen mal y no respondan a estas presiones terminarán, como los dinosaurios, siendo piezas de museo”. ¿Continuidad o cambio? Esa es la contradicción que hay que resolver y esto pasa necesariamente por la disposición de la comunidad universitaria de empoderarse de una nueva concepción y praxis del quehacer educativo, que forme para un nuevo contexto, donde es inviable continuar con un modelo educativo de carácter profesionalizante, cuando los cambios que la sociedad del siglo XXI demanda van más allá de los simples saberes. Se trata de una sociedad del conocimiento que demanda: investigación, innovación y emprendimiento, de manera tal que los saberes sean pertinentes, que sean producto de una renovación constante, de creatividad académica, que facilite procesos de inserción en un mundo que reclama nuevas formas de producción; nuevos modelos de formación, como también iniciativas novedosas para gestionar conocimiento, en fin; una universidad que debe replantear su quehacer en función de su calidad y sin lugar a dudas de su prestigio como máxima casa de estudios, respondiendo desde luego a las demandas de la sociedad, como bien público que es y como dice el Ingeniero Telémaco Talavera, Presidente del Consejo Nacional de la Educación Superior de Nicaragua "La universidad debe estar avocada con la sociedad, por ser esta misma el laboratorio más grande con que cuenta, estamos convirtiendo la escuela, la educación, la universidad de un círculo vicioso a un círculo virtuoso" y hace el llamado "debemos aumentar la masa crítica constructiva".

\section{Aprender para transformar}

La universidad tiene la enorme responsabilidad de contribuir a la transformación de nuestra sociedad, de nuestro país. No se puede, en este contexto contemporáneo de incertidumbres racionales, que desde las aulas de clase, desde la praxis educativa, basarse en los saberes para interpretar el mundo y su compleja realidad, porque sería estar en contra o a espaldas de la realidad que vivimos, desconocer la multi, la interdisciplinariedad de la misma signada por el avance vertiginoso del conocimiento que hoy está acompañado del surgimiento cada vez más abundante de disciplinas y subdisciplinas especializadas.

Hacer ciencia para el cambio estructural dentro y hacia afuera de la universidad, es una responsabilidad científica y académica que el alma mater debe asumir y el primer paso es: fomentar una cultura científica para enfrentar la fragmentación del conocimiento que, hoy en día, es un freno para la ciencia y para la formación de los profesionales de este nuevo siglo, pero, además de evitar la fragmentación, se debe facilitar procesos de innovación pedagógica y didáctica, para que el conocimiento y aprendizaje integrado de las ciencias trascienda los saberes, el academicismo y se inserten en una dinámica que posibilite vincular la ciencia con la realidad, con las necesidades que 
deben resolverse, para que los aprendizajes conduzcan a la formación de sólidas competencias de los estudiantes y futuros egresados para su incidencia en la trasformación productiva, económica, social, cultural y tecnológica de la sociedad.

Iniciativas novedosas que pueden ir sentando las bases de la multi e interdisciplinariedad sería que los docentes de un grupo determinado de cualquier asignatura, trabajen los planes didácticos en colectivo y no individual, (aunque cada docente tendrá su plan didáctico) esto permitiría tener a cuatro o cinco especialistas, depende de las asignaturas que lleva el grupo; reflexionar y planificar de manera integral que permita tener una visión de conjunto en el tratamiento al grupo, logrando la unidad del conocimiento, trascender los saberes y facilitar los aprendizajes de los estudiantes. El proceso de transformación curricular que vive actualmente la universidad, determinado por los retos que demanda el contexto contemporáneo, es un momento oportuno para reflexionar sobre la reintegración del saber, pero también la aplicación del mismo y desde luego, el valor que tiene la multi e interdisciplinariedad en la construcción de la universidad del siglo XXI. No es una tarea fácil, pero sí, una necesidad impostergable para la solución de los complejos problemas, tanto de las ciencias naturales, como de las ciencias sociales y humanas.

Edgar Morin insiste en que: "la universidad de hoy deberá centrase en la formación de un "ciudadano planetario", concentrado en pensar e interactuar con los nuevos problemas y epistemologías que plantea la sociedad actual, la universidad deberá formar para la vida, el sentido, la pasión y la transformación del mundo. Eso implica formar en tres dimensiones analíticas y transversales: a) los nuevos problemas universales/locales; b) las nuevas epistemologías que produce y entrega la sociedad contemporánea; y c) los "saberes civilizatorios" que constituyen las sociedades modernas, planetarias, integradas y complejas".

Todo estudiante de la universidad, igual que cualquier graduado, debe tener una formación interdisciplinar, además, sus conocimientos y sus praxis no deben estar basados en saberes puros y duros, sino más bien, en un proceso de construcción social del conocimiento que le permita forjarse como ser y como profesional preparado para un nuevo contexto que exige creatividad, innovación e ideas emprendedoras. ¿Acaso no es importante que un estudiante de economía profundice sobre los problemas que genera el cambio climático? ¿Qué un estudiante de trabajo social conozca sobre derecho procesal penal? ¿Qué un estudiante de agroindustria conozca a fondo sobre agricultura y desarrollo rural?, en fin, ya el conocimiento no puede ser unidisciplinar y muchos menos centrado en saberes como un círculo vicioso que debe romperse.

El valor estratégico que reviste la multi y la interdisciplinariedad para el desarrollo de la calidad educativa de la universidad, para una visión holística de su quehacer, que se encamine a materializar los aprendizajes para transformar la realidad, es fundamental y oportuno en el contexto actual signado por el aprendizaje permanente y ante nuevos paradigmas que demanda la educación superior. 


\section{Rompiendo esquemas para los aprendizajes a través de contenidos pertinentes}

La praxis educativa al romper con los saberes profesionalizantes, debe proponer aprendizajes significativos, como señala Ausebel, lo que implica en primer lugar la iniciativa creadora del docente para proponer contenidos educativos pertinentes, que permitan a estudiantes y docentes entrar al círculo virtuoso del aprender, del aprehender y del aprender a hacer y a resolver problemas, para encaminarse a innovar permanentemente desde la educación, que se traduce en una escuela que debe negar la anquilosada enseñanza vertical que más bien es un freno ante los nuevos retos que nos impone el conocimiento contemporáneo y que reclama esta sociedad, porque ambos: conocimiento y sociedad, unidos dialécticamente, están en permanente transformación.

¿Qué contenidos aprenden los estudiantes? ¿Para qué tipo de formación? ¿Están en correspondencia los objetivos propuestos con los contenidos? Estas interrogantes deben de reflexionarse, porque si desde la universidad hay todo un interés de forjar profesionales de calidad como lo demanda su modelo educativo, esto implica examinar el quehacer de la docencia aprendizaje, de forma tal que permita valorar la calidad de los aprendizajes, tanto en el proceso de formación del educando, como en su desempeño profesional, es decir; se ha venido poniendo a prueba la viabilidad del currículo.

Romper esquemas en los aprendizajes en este contexto en que nos desenvolvemos, implica hacer educación desde una praxis innovadora, donde cualquier asignatura no está escrita en piedra, donde los contenidos de la misma no son estáticos, todo lo contrario, están sometidos a una dinámica que impone el entorno y que la universidad debe enriquecer no sólo con la docencia, sino que también con ejes claves como la investigación y la extensión, pero impregnados de la innovación y el emprendimiento como dos factores estratégicos de la educación del siglo XXI y esenciales en todo modelo curricular que se precie de ser de avanzada.

La pertinencia en el aprendizaje de los contenidos es una acción innovadora, revestida de creatividad que el estudiante debe de comprender y asimilar porque parte de lo concreto, de lo tangible, de lo terrenal, del medio, de la vida, pero también el docente debe ser un facilitador comprometido con los aprendizajes. ¿Cómo hacer que los aprendizajes de historia sean asimilados por los estudiantes de todas las carreras y no solo de Ciencias Sociales? ¿Cómo empoderar a los estudiantes sobre la problemática del cambio climático y no solo a los de Ciencias Ambientales? ¿Y así sucesivamente, podemos ir considerando en las diferentes carreras que ofrece la universidad, la importancia que reviste la formación holística desde una asignatura hasta la comprensión y aplicación integral del currículo.

Es fundamental una revisión profunda de toda la cadena de valor que tiene como depositaria al aula de clase, donde debe garantizarse la calidad educativa, lo que implica creatividad: pasión, cultura científica, equilibrio pedagógico y enfoque dialéctico, con lo cual se debe garantizar la 
pertinencia de los contenidos que posibilite una praxis educativa de contexto, proyectada hacia el futuro y como antítesis de saberes anquilosados que ya no tienen razón de ser.

\section{La gestión del conocimiento y la pertinencia de los saberes}

El contexto contemporáneo, por una educación de calidad, exige romper con el academicismo, pero además; la universidad, por principio, debe rechazar la globalización del conocimiento, porque su objetivo es la uniformidad del pensamiento, para no problematizar, no cuestionar, no investigar, a que otros piensen por nosotros, a confirmar el fin de la historia, como lo ha querido imponer el filósofo norteamericano de origen japonés Francis Fukuyama. Frente a este panorama amenazador, donde la inteligencia puede ser arrastrada hacia el pensamiento hegemónico de los centros de poder mundial, es que se requiere que la universidad gestione conocimiento, lo que implica no sólo romper con una concepción globalizadora que uniforma epistémica y gnoseológicamente, sino que también con una concepción y una praxis pedagógica que reproduce saberes de forma mecánica en detrimento de los cambios estructurales que demanda la sociedad.

La universidad tiene un enorme reto en cuanto a gestionar conocimiento, no para reproducir el círculo vicioso del academicismo, sino más bien para contextualizarlo, hacerlo pertinente a través de una acción educativa innovadora, donde la investigación compartida; los colectivos científicos, las comunidades de aprendizaje sean claves en cuanto a elevar la calidad de la educación que apunte a una formación integral de los futuros profesionales, no obstante, los frutos de este esfuerzo deben estar impregnados de la multi e interdisciplinariedad, como condición sine qua nom en esta exigente sociedad del conocimiento en que se vive hoy.

La gestión del conocimiento es uno de los procesos fundamentales en la sociedad del conocimiento de este nuevo siglo. Se trata de conocer, dinamizar y aprovechar el beneficio colectivo que puede conseguirse de un trabajo colectivo que parte y considera el capital intelectual de los miembros de la organización, capital intelectual que debe estar al servicio del aprendizaje permanente, de la renovación de saberes, de la innovación que hoy se requiere para enfrentar las incertidumbres racionales y en este campo, la investigación es la clave para materializar la gestión del conocimiento, sobre todo en función del desarrollo humano, tan esencial en este siglo XXI, caracterizado por profundas desigualdades sociales; al respecto, Carmen Garcia Guadilla, al referirse a la pertinencia de las universidades en el nuevo contexto social, indica que: "El término que se utilice, pertinencia, extensión, responsabilidad social, no es el punto de interés, sino que la universidad debe responder al modelo de desarrollo humano y sustentable, donde el capital cultural de la ciencia y la tecnología sean aprovechados, no sólo en función de las necesidades del mercado, del capital económico, es fundamental reconciliar la innovación con la pertinencia social, la tradición con el cambio, la apertura al mundo con identidad propia, revalorar la subjetividad social y la dinámica cualitativa de la vida social". 
La cultura organizacional es clave para viabilizar procesos dentro de la universidad para gestionar conocimiento, es decir, darle el principal valor agregado al trabajo intelectual, a la investigación compartida, al trabajo en equipo, pero que al final tenga beneficios tangibles para la sociedad.

La utilización del conocimiento para fines educativos, sociales, productivos, etc. sólo era posible formando equipos de trabajo de diferentes disciplinas e ir construyendo, gestionando conocimiento a partir de trabajos de campo, diagnósticos, entrevistas, observación, estudio individual, estudio en grupo, etc.

La gestión del conocimiento en la universidad, debe llevar a desarrollar esfuerzos de investigación donde, en su momento, los docentes preparen material que sea de consulta de los estudiantes, también los productos de la JUDC deben convertirse en material de consulta para los estudiantes de las diferentes carreras, se trata de generar resultados tangibles en materia de producción científica, compartirla y materializarla como expresión de una universidad que rompe con esquemas obsoletos de enseñanza y se apunta por aprendizajes significativos, expresión de una forma innovadora y creativa de hacer educación y signo inequívoco de los nuevos tiempos que deben ser interpretados por la universidad.

Gestionar conocimiento es construir nuevos conocimientos, de innovar, de no imitar, de no copiar, de no cortar o pegar, que se ha vuelto hoy toda una cultura o contracultura. Por ejemplo Internet es un valioso recurso, pero a mi juicio es sólo un inmenso banco de información, que nos puede volver dependientes y que dejemos de pensar, de crear, por lo tanto, el primer paso es construir nuevos conocimientos, gestionar nuevos conocimientos a partir de la realidad y hacerlos realmente sostenibles, que significa materializarlos y que reflejen resultados cualitativos.

Virtuosidad real y su aporte al aprendizaje de contexto, el caso de las TIC.

Toda acción educativa, en este mundo contemporáneo, no puede obviar las tecnología de la información y la comunicación, TIC, precisamente porque son hijas de la sociedad del conocimiento y de la información en que vivimos y de la cual, la universidad no puede autoexcluirse tomando en cuenta que el mundo virtual es una realidad inobjetable y que la relación dialéctica de lo virtual y lo real, en el campo de los aprendizajes; debe ser manejado con el equilibrio pedagógico necesario para que en la relación docencia y tecnología, tengamos la mayor productividad posible en función de aprendizajes significativos que se traduzcan en una formación pertinente y de calidad; al respecto, Dolors Reig nos indica lo siguiente, sobre información recabada con jóvenes, sobre el significado de las TIC en sus vidas: "En el contexto tecnocultural actual, la relación entre el mundo real y el mundo virtual no puede entenderse como un vínculo entre dos mundos independientes y separados, que eventualmente coinciden en un punto, sino como una cinta de Moebius, donde no existe un adentro y un fuera, y donde es imposible identificar límites entre ambos. Para las nuevas generaciones, cada vez más, la vida digital se fusiona con su domesticidad como un elemento más de la naturaleza. En esta naturalización de la vida digital, los aprendizajes provenientes de ese 
entorno se mencionan con asiduidad, no solo porque se los pregunta explícitamente sino porque la referencia a Internet sale del modo más espontáneo entre los entrevistados”.

La misma Dolors Reig, nos señala otra novedad en el aprendizaje desde lo virtual, cuando dice: "A grandes rasgos, se trata de que profesores y alumnos desarrollen y compartan competencias en torno a tres conjuntos de siglas: TIC (Tecnologías de la Información y la Comunicación), TAC (Tecnologías del Aprendizaje y el Conocimiento) y TEP (Tecnologías del Empoderamiento y la Participación)."

El mundo virtual en la universidad, debe considerarse, en general, como parte de la innovación educativa, como eje transversal del currículo y en particular, en el proceso de aprendizaje en el aula; innovación que implica el aprovechamiento didáctico de las TIC, para que creativamente se utilicen, sin abusar de ellas, porque al final de cuentas, la relación horizontal: docente -estudiante es lo que determina la esencia de la educación, pero también, el aprovechamiento de las tecnologías, vistas desde la perspectiva de la inmensa cantidad de información que fluye por las redes, debe ser utilizada productiva y académicamente en el sentido de contextualizarla y darle pertinencia, siempre y cuando se traduzca en conocimiento objetivo para un aprendizaje permanente, que sea factor de cambio, que transforme el medio y que trasforme al ser. Sobre la relación horizontal docente-estudiante, es importante citar a Joaquín Brunner, que nos indica: "No debe cometerse el error de imaginar que el cambio educacional será guiado por las nuevas tecnologías de información y comunicación, por poderosas que estas sean. La educación es mucho más que sus soportes tecnológicos; encarna un principio formativo, es una tarea social y cultural que, cualesquiera sean las trasformaciones que experimente, seguirá dependiendo, ante todo, de sus componentes humanos, ideales y valóricos".

La virtuosidad para que responda al encargo social de la universidad, debe ser real, porque el peor error en que se puede caer, es considerar que lo puramente virtual, la cultura de copiar y enseñar y la cultura de copiar y pegar, tanto para el ejercicio de la docencia, como para el discente sea lo mejor, lo más práctico, esto más bien genera un subdesarrollo mental, una colonización virtual que la aprovechan los centros hegemónicos de poder, para que el pensamiento genuino no florezca y en este siglo XXI, la universidad, sobre todo en los países subdesarrollados, debe hacer aportes sustanciales al desarrollo, porque el crecimiento de un país, hoy lo determina el conocimiento, la formación, la educación de una sociedad; al respecto, Manuel Castell nos dice: “¿Qué sistema de comunicación es entonces el que, en contraste con la experiencia histórica previa, genera virtualidad real? Es un sistema en el que la misma realidad (esto es, la existencia material/simbólica de la gente) es capturada por completo, sumergida de lleno en un escenario de imágenes virtuales, en el mundo de hacer creer, en el que las apariencias no están sólo en la pantalla a través de la cual se comunica la experiencia, sino que se convierte en la experiencia. Todos los mensajes de toda clase quedan encerrados en el medio, porque éste se ha vuelto tan abarcador, tan diversificado, tan maleable, que absorbe en el mismo texto multimedia el 
conjunto de la experiencia humana, pasada, presente y futura”. La virtuosidad real, aprovechada metodológicamente, debe asegurar la participación de los educandos en la acción educativa que se genera en el aula y esto tiene un nombre: participación, o en palabras de Paulo Freire, pedagogía de la pregunta, el maestro decía: "Es necesario desarrollar una pedagogía de la pregunta. Siempre estamos escuchando una pedagogía de la respuesta. Los profesores contestan a preguntas que los alumnos no ha hecho". La ventaja comparativa de ser nativos digitales, le permite a los actuales estudiantes insertarse sin traumas en el mundo de las nuevas tecnologías, de lo que se trata es de que se les faciliten los procesos para que el provecho en el uso de las mismas sea sustancial, para que sea de carácter cualitativo, para que se prolongue en el tiempo de manera indefinida, que sean parte de la universidad de la vida, porque estas tecnologías: el mundo virtual, se quedó para siempre, pero cada día con insospechados cambios, de ahí que, la innovación desde las aulas universitarias debe ser permanente, porque de lo contrario, la post universidad puede ganar la partida, es decir, nuevas instituciones que interpreten los signos de los tiempos y que respondan a los retos y demandas de las nuevas generaciones.

El mundo virtual en la Educación Superior, aunque representa una ventaja comparativa importante, por la conectividad permanente de la comunidad universitaria, es una mayúscula responsabilidad, porque docentes y estudiantes deben utilizarlo creativa, pedagógica y didácticamente dentro y fuera del aula, que permita gestionar conocimiento y combinarlo entre lo abstracto y lo concreto para que se traduzca en resultados tangibles en beneficio de la ciencia y la sociedad.

¿Cómo valorar el aporte de las TIC a la educación? Esto solo es posible a través de procesos de evaluación y autoevaluación que refleje la asimilación de los aprendizajes y su puesta en práctica a partir de diferentes estrategias a saber: uso responsable y discriminatorio de la información que fluye por internet, formación de comunidades de aprendizaje que se convocan por la red, consultas a blogs, debates por Sky, video conferencias, uso responsable de facebook, pruebas sistemáticas u otros tipos de exámenes, etc.

La rica experiencia evaluativa que se derive de la aplicación de las TIC a la misma, deberá ser un insumo valioso para la universidad, en el sentido de generar propuestas innovadoras que faciliten procesos de evaluación y autoevaluación que vayan más allá de los exámenes tradicionales, es decir, la relación de lo virtual y lo real que procure aprendizajes significativos, a partir de su aplicación al entorno.

\section{La JUDC y el círculo virtuoso del innovar y el emprender}

La innovación y el emprendimiento, deben ser las banderas del siglo XXI, lo cual es el reflejo de un mundo y una sociedad de cambios vertiginosos e insospechados, en este sentido; el cambio de paradigma desde la perspectiva curricular de la universidad no puede esperar, es un sí o sí académico, científico y técnico, porque esta sociedad del conocimiento y de la información, con 
su acelerado avance nos obliga a reflexionar, a cuestionarnos sobre qué tipo de educación hay que proponer, para qué tipo de desarrollo. También, en este cambio de época que vivimos, el conocimiento por sí solo no es nada, se trata de saber utilizarlo, darle viabilidad y eso solo es posible desde una praxis educativa innovadora, encaminada al emprendimiento que tenga en la investigación la punta de lanza de los aprendizajes significativos de los estudiantes, para que el conocimiento dé respuesta a su formación integral y al desarrollo de la sociedad en los aspectos técnicos, científicos, productivos, ambientales, económicos y culturales.

En esta universidad del siglo XXI, en el que innovar y emprender se vuelven fundamentales, la JUDC es la plataforma ideal para volcar todas las iniciativas creadoras y el talento de los estudiantes para demostrar que el espíritu innovador y emprendedor lo andan consigo, que la pelota está en la cancha de la academia, que necesita repensar el currículo y ajustarlo a las nuevas realidades que requiere de respuestas pertinentes. El compromiso en la formación de profesionales que se correspondan con los nuevos tiempos, solo será posible desde una pedagogía innovadora, en que no veamos la ciencia como algo acabado, sino en permanente construcción, como un aprendizaje permanente de todos, que nos lleve del yo empírico al sujeto epistémico, que se traduzca en un quehacer innovador, emprendedor, como ya lo es la JUDC. De lo que se trata es que esta rica experiencia que cada año muestra sus progresos, en información, investigación y proyectos esté a disposición de la sociedad en sus diferentes expresiones (Gobierno, Alcaldías, sector privado, etc.) y desde luego, la JUDC es una factor de cambio individual, porque nuestros estudiantes están construyendo sus propios proyectos de vida y desde luego que también nacional, porque la universidad está en función de los grandes retos de la nación; de ahí la vigencia en lo antes señalado del Plan Nacional de Desarrollo 2012-2016, cuando indica: "En Nicaragua, la Ciencia, la Tecnología, la Innovación y el Emprendedurismo serán motores centrales para la transformación económica y social en el mediano y largo plazo. Las innovaciones tecnológicas producto del desarrollo del conocimiento, generan mayor competitividad de las empresas y los países, agregando valor a sus productos y servicios".

El modelo educativo pensado y formulado desde el presente, debe estar orientado hacía el futuro, por la naturaleza misma del conocimiento contemporáneo que conduce a una educación para toda la vida y que obliga a la universidad a trabajar en la línea de los paradigmas del aprender a aprender, aprender a emprender, aprender a resolver problemas y aprender a convivir en mundo más humano y más solidario; ¿Acaso la JUDC, no está en sintonía con esos paradigmas planteados en su momento por la UNESCO a través de la comisión Delors, en aquél histórico documento "la educación encierra un tesoro", que hoy sigue teniendo vigencia”? eso implica, que el graduado universitario debe estar dotado de las destrezas científico- técnicas necesarias y de los valores como ser humano para enfrentar los retos de este mundo globalizado y del conocimiento en permanente transformación, esto no exime a los docentes como facilitadores de un quehacer educativo que requiere replanteamientos serios y de una obligatoria y permanente cultura científica. 
El reflejo de una JUDC que cada año dé saltos de calidad, es consecuencia de una educación universitaria que cada día toma conciencia que su quehacer pedagógico está en la investigación, que los aprendizajes se sustenten en ella; que es la punta de lanza para forjar profesionales, que al volcar sus conocimientos a la economía del país, a la producción o a cualquier campo ligado al desarrollo nacional; tiene que incidir en la productividad, en los niveles de producción que han sido un talón de Aquiles que ha frenado nuestro desarrollo, por lo que el complemento de estos aprendizajes, además del acervo de conocimientos tecnológicos, es una cultura empresarial que conlleve a la creación de negocios, que generen productos de calidad competitivos que reflejen una cultura emprendedora.

En definitiva, la cultura investigativa debe marcar el presente y el futuro de la universidad y la JUDC como parte de esa cultura es la plataforma que sienta las bases innovadoras y emprendedoras, como elementos claves de este nuevo siglo y que el Alma Mater debe potenciar como respuesta pedagógica, técnica, y científica, en función de la formación integral de los estudiantes y del desarrollo local y nacional.

\section{La universidad y la formación de valores, un factor clave para humanizar la ciencia}

Sin desarrollo científico, tecnológico y cultural es imposible el desarrollo social y nacional, no obstante; la ciencia y la cultura deben tener como parámetro fundamental la formación de valores que implica la construcción del ser en su sentido más integral, lo que, toda propuesta educativa, toda concepción y propuesta curricular, debe ser transversalizada por el paradigma ontológico-axiológico como condición sine qua non en la forja de profesionales que contribuyan a la construcción de una sociedad más justa, trasparente y con identidad de nación.

El actual mundo globalizado se caracteriza por enormes desigualdades económicas y sociales, fruto de un modelo capitalista concentrador de la riqueza y generador de pobreza; ante esta realidad, las universidades deben ser claves en la reducción de las asimetrías y la única forma es forjando profesionales dotados de ciencia y de conciencia, para contribuir a darle un rostro más humano a la sociedad y sobre todo en favor de los sectores más vulnerables que no dejan de contribuir con su trabajo al desarrollo de una nación; en este sentido, es importante indicar que hoy, el PIB, no es garantía del bienestar ciudadano, se requiere de otros instrumentos como la herramienta del Índice de Progreso Social (IPS), la cual define el éxito de una sociedad de acuerdo a su capacidad para satisfacer las necesidades básicas, generar las condiciones para mejorar la calidad de vida, proteger el ambiente, y ofrecer las oportunidades para que sus ciudadanos alcancen su pleno potencial, ahí está el papel de la educación superior, encontrar la opciones para buscar las respuestas pertinentes que demanda la sociedad, la nación.

La formación de valores trasciende todo, es la base para que las personas actuemos privilegiando lo humano, dignificando y visibilizando al ser como el eje y punto de referencia para cualquier cambio. 
Los valores bien cimentados desde la universidad, debe ser una garantía para que sus egresados contribuyan al bienestar de la sociedad y a la lucha constante contra las asimetrías económicas y sociales, la corrupción y otras formas de agresión a la sociedad que es el reflejo de la deshumanización, al respecto, Felipe Abundis de León, de la universidad de la Rioja señala que: "El momento actual es un buen momento para revisar la cuestión de los propósitos públicos de la Educación Superior. Consideramos que si los graduados actuales están llamados a ser una fuerza positiva en el mundo, necesitan no sólo poseer conocimientos y capacidades intelectuales, sino también verse a sí mismos como miembros de una comunidad, como individuos con una responsabilidad para contribuir a sus comunidades. Deben ser capaces de actuar para el bien común y hacerlo efectivamente”.

La universidad pública, como parte de la superestructura de la sociedad, debe hacer prevalecer la importancia estratégica de los valores, aprovechando la autonomía relativa o absoluta de la superestructura, porque no es necesaria la modernidad económica o un desarrollo excepcional de la infraestructura para que se produzcan cambios en la conciencia, o para romper con el flagelo de la corrupción u otros males endémicos que afectan a la sociedad; la universidad está en capacidad de contribuir al desarrollo social y nacional y la formación de valores y la puesta en práctica de los mismos son esenciales, necesarios en el presente siglo.

\section{Repensar el aula}

En la sociedad del conocimiento y de la información en que vivimos, la educación requiere un replanteamiento serio, dado que el conocimiento está entrando en la obsolescencia de forma vertiginosa, pero también los educandos del siglo XXI son una generación digital que depende mucho de lo virtual. En ese sentido, se vuelve una imperiosa necesidad el acompañarlos para facilitarles los aprendizajes con significatividad y pertinencia, de forma tal que la praxis educativa no se vuelva un círculo vicioso, sino más bien de carácter sustancial con resultados cualitativos y que por el contexto en que se desenvuelve, forme para la vida, tal y como dice Paulo Freire, "por medio de la misma vida".

El espacio inmediato formal donde se practica el ejercicio educativo es el aula. Ahí se da el encuentro cotidiano de maestros y alumnos para desarrollar el aprendizaje, que como un proceso dialéctico del conocimiento, deberá estar siempre en permanente construcción, apuntando a lo epistémico-ontológico, pero sin desconectarse de la realidad. De ese modo, juntos, maestros y alumnos, construyan propuestas novedosas y que el aula sea un círculo virtuoso como lo demanda el contexto contemporáneo.

Yo señalaba en un escrito anterior, que de nosotros los docentes dependía que el aula entrara en crisis. Esto lo señalaba a propósito de varios factores, uno de ellos es la sociedad del conocimiento en que vivimos hoy en día, y en particular del mundo virtual que forma parte de los útiles escolares 
de los estudiantes. Estos están enfrentando un proceso de transición inevitable entre la enciclopedia y la información global, empoderándose de esta última sin ningún obstáculo, incluso, este tipo de ventaja cognoscitiva, los puede llevar a romper con el conocimiento fragmentado antes que los docentes, que seguimos -en el caso de la educación superior- sumergidos en compartimientos estancos, es decir, desde nuestros departamentos como pozos tiradores, en una tercia académica y "científica", de dolorosas consecuencias presentes y futuras para la educación.

En todos los niveles de educación se debe repensar el aula, desde el preescolar, donde se sientan las bases de la formación, hasta la universidad, que es el producto final. Por eso, los esfuerzos que se realizan en materia educativa, en nuestro país, para establecer un vínculo orgánico entre los diferentes subsistemas, constituye un paso estratégico de invaluable importancia, encaminado a dar esos saltos de calidad que tanto necesitamos.

Repensar el aula pasa necesariamente por desarrollar los procesos de innovación y emprendimiento desde la infancia, hasta la formación técnica y profesional, porque como señala Ken Robinson : "Los niños arriesgan, improvisan, no tienen miedo a equivocarse; y no es que equivocarse sea igual a creatividad, pero sí está claro que no puedes innovar si no estás dispuesto a equivocarte, y los adultos penalizamos el error, lo estigmatizamos en la escuela y en la educación, y así es como los niños se alejan de sus capacidades creativas”. Igualmente, para el caso de la educación superior, como sostiene la UNESCO: "En una época en que ya no se puede aplicar más la ecuación "título=trabajo, se espera que este nivel de educación produzca egresados que no sólo puedan ser buscadores de trabajo, sino también empresarios de éxito y creadores de empleo”.

En definitiva, es una necesidad apremiante reflexionar y actuar ante los retos presentes y futuros que debe enfrentar la educación a todos los niveles y repensar el aula implica, en términos estratégicos, el desarrollo de aprendizajes de calidad a lo largo de toda la vida, como indica la declaración de Incheon, sólo de esa manera nos encaminaremos por la senda del crecimiento y del desarrollo integral sostenible, tanto humano como social y económico.

\section{CONCLUSIÓN}

El reto que la universidad tiene en este nuevo siglo es mayúsculo, es un serio compromiso por hacer valer la ciencia y la cultura en un nuevo contexto, lo que equivale a viabilizar y aplicar el conocimiento de manera pertinente en función de la sociedad, por eso es imprescindible la ruptura con los saberes profesionalizantes, porque de lo contrario la contribución de la universidad con la formación integral del ser humano y los cambios estructurales en la sociedad serán imposibles, con

lo cual, la máxima casa de estudios estaría de espaldas a una realidad signada por el aprendizaje permanente como lo demanda el contexto contemporáneo. 
Hoy más que nunca, la universidad debe abocarse a la actualización permanente del currículo para que su quehacer esté a la altura de los tiempos, lo que equivale a ofrecer una educación con pertinencia, donde sus ejes fundamentales: docencia, investigación y extensión, en una unidad dialéctica, contribuyan a la formación integral de las presentes y futuras generaciones de profesionales de nuestro país. La ruptura con los saberes profesionalizantes, es enrumbarse por la senda del circulo virtuoso, que equivale a una nueva forma de hacer educación, donde la curiosidad científica, la observación, el ensayo y el error, lo que no está resuelto; y el desarrollo de competencias cognitivas, que sumado a la cultura de innovar y emprender, deben sentar las bases de una universidad consciente de su papel para los cambios paradigmáticos que demanda esta sociedad del aprendizaje permanente que hoy vivimos.

\section{REFERENCIAS BIBLIOGRÁFICAS}

Bernheim, C. (2010). La educación permanente y su impacto en la educación superior. Revista Iberoamericana de Educación Superior, Vol. 1 núm. 1.

Brunner, J., \& Braslavki, C. (s.f.). Las Políticas Educativas Frente a la Revolución Tecnológica en un Mundo de Interdependencias Crecientes y Parciales. Obtenido de UNESCO: http://www. unesco.org/new/es/unesco/

Castañeda, D. O. (2017). Monografías.com. Obtenido de http://www.monografias.com/trabajos97/ filosofia-rene-descartes/filosofia-rene- descartes.shtml\#ixzz4bKiMuRFH

Castells, M. (1997). La era de la información. Economía, cultura y sociedad. Obtenido de Alianza: http://apolo.uji.es/Emilio/IS/T4-3.html

CNU. (16 de febrero de 2017). Consejo Nacional de Universidades. Obtenido de Retos de la Universidad Contemporánea: http://www.cnu.edu.ni/retos-de-la-universidad- contemporanea/

Coombs, P. (s.f.). las universidades en el siglo XXI.

Cultura, C. (Octubre de 2012). Mejores 20 frases de Paulo Freire. Obtenido de https://canalcultura. org/2012/10/01/mejores-20-frases-de-paulo-freire-educacion/

León, F. A. (2012). Dialnet. Obtenido de Universidad y formación de valores en un contexto posmoderno: https://dialnet.unirioja.es/servlet/articulo? codigo $=4034807$

Martín, A. R. (2017). Lifeder.com. Obtenido de Las 37 Mejores Frases de Isaac Newton: https:// www.lifeder.com/frases-de-isaac-newton/

Martín, A. R. (2017). Lifeder.com. Obtenido de frases de Arquimedes: https://www.lifeder.com/ frases-de-arquimedes/

Nacional, G. d. (2012). Plan Nacional de Desarrollo Humano. Obtenido de http://www.pndh.gob. ni/

Neves, D. M. (s.f.). TENDENCIAS Y RETOS DE LAS UNIVERSIDADES PÚBLICAS ENAMÉRICA LATINA ANTE LOS NUEVOS PARADIGMAS DE PERTINENCIA Y RESPONSABILIDAD SOCIAL. Obtenido de Universidad Pedagógica de México: http://actacientifica.servicioit.cl/ biblioteca/gt/GT17/GT17_deSierraNeves.pdf.

Reig, D. (2016). La virtualización de la educación superior en América Latina: entre tendencias y paradigmas. TIC-TAC-TEP: las siglas del aprendizaje aumentado. 
Robinson, K. (s.f.). La escuela mata la creatividad. Obtenido de Ken Robinson: ¿ "La escuela mata la creatividad"? http://www.lavanguardia.com/estilos-de- vida/20120203/54247867713/ la-escuela-mata-la-creatividad.html.

Roveda, A. (2010). Los principios fundamentales de la educación del siglo XXI. Conversaciones con Edgar Morin. Javeriana.

Sophimania. (2017). 6 alucinantes predicciones de Ray Kurzweil, el futurista de Google. Obtenido de -https://www.sophimania.pe/tecnologia/redes-sociales/6-alucinantes

UNESCO. (1995). UNESCO. Obtenido de Documento de Política para el Cambio y Desarrollo en la Educación Superior: http://unesdoc.unesco.org/images/0009/000989/098992s.pdf 